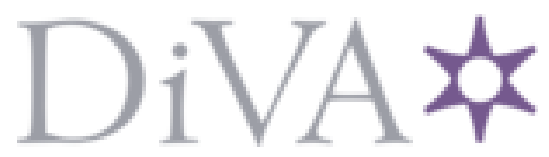

http://www.diva-portal.org

This is the published version of a paper published in Physical Review A. Atomic, Molecular, and Optical Physics.

Citation for the original published paper (version of record):

Linusson, P., Fritzsche, S., Eland, J., Hedin, L., Karlsson, L. et al. (2011)

Double ionization of atomic cadmium.

Physical Review A. Atomic, Molecular, and Optical Physics, 83(2): 023424

http://dx.doi.org/10.1103/PhysRevA.83.023424

Access to the published version may require subscription.

N.B. When citing this work, cite the original published paper.

Permanent link to this version:

http://urn.kb.se/resolve?urn=urn:nbn:se:uu:diva- 149573 
PHYSICAL REVIEW A 83, 023424 (2011)

\title{
Double ionization of atomic cadmium
}

\author{
P. Linusson \\ Department of Physics, Stockholm University, AlbaNova University Centre, SE-10691 Stockholm, Sweden \\ S. Fritzsche \\ Department of Physics, P. O. Box 3000, FIN-3004 University of Oulu, Finland \\ GSI Helmholtzzentrum für Schwerionenforschung D-64291 Darmstadt, Germany and Frankfurt Institute for Advanced Studies, \\ D-60438 Frankfurt am Main, Germany \\ J. H. D. Eland \\ Department of Chemistry, Physical and Theoretical Chemistry Laboratory, Oxford University, South Parks Road, Oxford OX1 3QZ, \\ United Kingdom and Department of Physics and Astronomy, Uppsala University, Box 516, SE-75120 Uppsala, Sweden \\ L. Hedin, L. Karlsson, and R. Feifel \\ Department of Physics and Astronomy, Uppsala University, Box 516, SE-75120 Uppsala, Sweden
}

(Received 24 November 2010; published 28 February 2011)

\begin{abstract}
We have recorded the double photoionization spectrum of atomic $\mathrm{Cd}$ at four different photon energies in the range 40-200 eV. The main channel is single ionization and subsequent decay of excited $\mathrm{Cd}^{+}$states, some involving Coster-Kronig processes, whereas direct double ionization is found to be weak. The decay of the excited $\mathrm{Cd}^{+}$states shows a strong selectivity, related to the configuration of the final state. Double ionization leading to the $\mathrm{Cd}^{2+}$ ground state is investigated in some detail and is found to proceed mainly through ionization and decay of $4 d$ correlation satellites. The most prominent autoionization peaks have been identified with the aid of quantum-mechanical calculations.
\end{abstract}

DOI: 10.1103/PhysRevA.83.023424

PACS number(s): 32.80.Fb, 32.80.Zb, 34.50.Gb

\section{INTRODUCTION}

Double ionization of atomic cadmium was first studied by Cairns et al. [1], who reported the partial cross section for production of $\mathrm{Cd}^{2+}$ and also extended the previous singleionization measurements of Berkowitz and Lifshitz [2] over a wider photon energy range. The conventional photoelectron spectrum of $\mathrm{Cd}$ at $\mathrm{HeI}$ photon energies was recorded by Süzer and Shirley and was found to contain satellite peaks in addition to the main $5 s$ and $4 d$ lines; these were attributed to configuration interaction (CI) in the initial state [3]. It was later shown by Hansen, however, that knowledge of the expansion coefficients in the initial state alone could not explain the intensities of the satellite states [4]. A subsequent study of the absorption spectrum of $\mathrm{Cd}$ revealed resonances that correspond to two-electron excitations, which could instead be attributed primarily to interaction in the final state [5]. A more recent study of the absorption spectrum of $\mathrm{Cd}^{+}$also showed resonances that were tentatively assigned to two-electron excitations [6].

Subsequent work on photoionization of $\mathrm{Cd}$ in the ultraviolet (UV) spectral region focused on the $4 d$ ionization cross section [7] and the effect of autoionization on $5 s$ and $4 d$ photoemission [8,9]. The x-ray-excited inner-shell photoelectron (XPS) and Auger spectra of Cd involving a $4 p$ hole were found to be very unusual [10,11], as were the XPS and x-ray emission spectra (XES) of other elements around $Z=50$ [10,12-14]. In the XPS spectrum of cadmium a broad asymmetric single peak was found for $4 p^{-1}$ ionization instead of the doublet expected from a one-particle picture, akin to the case of atomic Xe, where only a broad continuum could be seen in place of a $4 p_{1 / 2}$ peak. Lundqvist and Wendin [15] and, later, Wendin and Ohno [16] discussed the anomalous line shapes in terms of a very strong interaction between the $4 p$ hole and $4 d^{-2} n(\epsilon) f$ continuum and bound states. Considerable theoretical effort has since then been devoted to further explain the puzzling experimental results; for a recent review on this topic see, e.g., Ref. [17].

Apart from the early study by Cairns et al. [1], we are aware of the work on the single-photon double ionization processes in cadmium carried out by Holland et al. [18], who measured the double-ionization cross sections of $\mathrm{Zn}$, $\mathrm{Cd}$, and $\mathrm{Hg}$ near threshold in more detail. In this previous study the authors assumed that the double-ionization process is mainly direct (i.e., simultaneous emission of two electrons) at the photon energies chosen. Mansfield and Murnane [19] classified several lines from autoionization leading to doubly ionized cadmium in an analysis of the ejected-electron spectrum of cadmium excited by electron impact [20].

In this work we report angle-integrated single-photondouble ionization electron spectra of $\mathrm{Cd}$ measured at photon energies both below and above the $4 p$ threshold. Preliminary experiments by one of us on double photoionization of $\mathrm{Cd}$ using $\mathrm{He}$ light were reported previously [21]; this article expands and interprets those results. A multielectron coincidence technique has been used to reveal the complete electron pair energy distributions, which give important information on the double-ionization processes involved. New calculations have been undertaken to clarify the main mechanism for formation of the $\mathrm{Cd}^{2+}$ ground state. 


\section{EXPERIMENTAL DETAILS}

Two sources of ionizing radiation have been used for the present work. For the data recorded at 40.814 and $48.37 \mathrm{eV}$ photon energy a pulsed helium discharge lamp was used, which produces approximately 10-ns-long light pulses at a repetition rate of a few kilohertz [22]. All other experiments were carried out at beam line U49/2-PGM-2 [23] at the BESSY-II storage ring in Berlin, Germany, which provides light pulses of approximately 30 ps duration every $800.5 \mathrm{~ns}$.

The experiments were carried out using two different timeof-flight (TOF) magnetic bottle electron spectrometers for data collection. These instruments are capable of coincidence measurements and have been described in detail before [22,24-26]. In both cases they were modified for the present work to allow for evaporation of metallic cadmium by replacing the gas-needle setup by a small vacuum furnace, as in the work of Ref. [27]. Briefly, cadmium was evaporated from a small stainless steel cylinder and let into the interaction region through a protruding copper tube aligned to intersect the light beam. Heating was applied by Thermocoax cables wound around the metal cylinder and was controlled so a temperature of $\sim 220^{\circ} \mathrm{C}$ was reached. Close to the interaction region a conically shaped strong permanent magnet is located. When ionization takes place the divergent magnetic field lines force electrons emitted at nearly any angle to follow spiralling trajectories into the flight tube, which was approximately $5.5 \mathrm{~m}$ long in the experiments that used the helium lamp and $2.2 \mathrm{~m}$ long in the experiments carried out at the synchrotron. The flight tube is equipped with a solenoid to produce a weak magnetic field, guiding the electrons to an MCP detector positioned at the other end. Electron arrival times at the detector are recorded relative to the capillary helium discharge and relative to the synchrotron ring cycle, respectively. Conversion of the electron flight times to energy was calibrated using the well-known Xe Auger spectrum [28] for the synchrotron radiation experiments and using the well-known $\mathrm{O}_{2}$ outer valence photoelectron spectrum [29] for the experiments carried out using the helium lamp, respectively. The numerical energy resolution of single electrons was approximately $1 / 50$ for kinetic energies $>1 \mathrm{eV}$ in the experiments using the shorter apparatus and $1 / 100$ in the experiments that used the longer apparatus.

\section{THEORETICAL DETAILS}

To understand the double ionization of vaporized cadmium, photoionization and Auger calculations have been performed for this atom with a neutral ground-state configuration of $4 d^{10} 5 s^{2}$ by applying multiconfiguration Dirac-Fock (MCDF) wave functions. For open-shell structures, and especially open $d$ shells, the MCDF method has been found to be a very versatile tool to calculate ionization cross sections and rates due to various excitation and decay processes [30]. In this method [31], an atomic state is approximated by a linear combination of so-called configuration state functions (CSF) of the same symmetry

$$
\left|\psi_{\alpha}(P J M)\right\rangle=\sum_{r=1}^{n_{c}} c_{r}(\alpha)\left|\gamma_{r} P J M\right\rangle
$$

where $n_{c}$ is the number of CSF and $\left\{c_{r}(\alpha)\right\}$ the representation of the state in the given many-electron basis. In ansatz (1), moreover, $\gamma_{r}$ represents the occupation of the atomic shells as well as all further quantum numbers from the coupling of these shells as required for a unique specification of the $N$-electron basis. As in most standard computations, the CSF were constructed as antisymmetrized products of a common set of orthonormal orbitals and were optimized on the basis of the Dirac-Coulomb Hamiltonian. Further relativistic contributions to the representation $\left\{c_{r}(\alpha)\right\}$ of the atomic states have been added and helped improve the low-lying level structure and transition amplitudes. For mid- $Z$ elements, such as cadmium, especially the Breit interaction becomes noticable in the lowlying level structure of the nearly neutral ions while further radiative corrections remain negligible as long as no deep core shells are involved in the process [32].

Often the main limitations of the MCDF model arise from the omission of some electronic correlations due to a restricted size of the wave function expansion. This applies especially to the excitation and ionization processes that result in an open $d$ shell and becomes more pronounced if additional $4 d$ or $5 s$ electrons are excited. For neutral and singly ionized cadmium, such complex shell structures arise after the photoionization of a $4 d$ electron, which is further accompanied by an $5 s \rightarrow(6 s+7 s+5 p+5 d)$ shakeup. For the description of the neutral atoms as well as the singly and doubly charged ions, all atomic state functions have been generated by means of the GRASP92 code [33] and utilized within the RATIP program [34] in order to evaluate all the required amplitudes for modeling the ionization paths of cadmium. Apart from the (nominal) $4 d^{10} 5 s^{2}, 4 d^{10} 5 s$, and $4 d^{10}$ reference configuration of these three charge states, all single and double excitations into the $5 p, 5 d, 6 s, 6 p$, and $7 s$ shells were taken into account to allow for a proper flexibility in the description of the (photo-) ionization and subsequent decay processes. While the discussion below is based mainly on a single-configuration notation, configuration mixing has been taken into account in the representation of the wave functions, and in several cases quite strong mixing is observed. In addition, several of the state vectors have been transformed into an LSJ coupling scheme [35] to better understand and extract the ionization paths. For open $d$-shell structure, however, the size of the wave function expansions increase so rapidly that no attempt was made to include further virtual excitations in the construction of the wave functions. It was also impossible to monitor in detail how the convergence of the cross sections and rates depends on a further increase of $n_{c}$ in expansion (1).

Using the wave functions of the $4 d^{10} 5 s^{2}{ }^{1} S_{0}$ ground state of neutral cadmium, the $4 d$ photoionization cross sections

$$
\sigma\left(J_{f} P_{f}\right)=\frac{4 \pi^{2} \alpha \omega}{3\left(2 J_{g}+1\right)} \sum_{\kappa_{c}, J_{t}}\left|D\left(\omega ; J_{f} P_{f}, \epsilon \kappa_{c}: J_{t} P_{t}\right)\right|^{2},
$$

for the various final states $\left|\psi_{f}\right\rangle=\left|\psi\left(J_{f} P_{f}\right)\right\rangle$ of the photoion from the $4 d^{9}\left(5 s^{2}+5 s 5 p+5 s 5 d+\ldots\right)$ configurations have been calculated within the dipole approximation. In these cross sections, a summation has to be performed over all possible scattering states of the final system "photoion + electron" and where $\alpha$ denotes the fine-structure constant, $\hbar \omega$ the photon 
energy, and $J_{g}$ the total angular momentum of the initial (ground) level $\left|\psi_{g}\right\rangle \equiv\left|\psi\left(J_{g} P_{g}\right)\right\rangle$ of the atom. A summation runs over all possible scattering states of the atom. In particular, all partial waves of the photoelectron with kinetic energy $\epsilon=$ $E_{g}+\omega-E_{f}$ as well as over the continuum states $\left|\psi_{t}\right\rangle \equiv$ $\left|J_{f} P_{f}, \epsilon \kappa_{c}: J_{t} P_{t}\right\rangle$ with total angular momentum $J_{t}=1$ and odd parity were taken into account. Moreover, the relaxation of the bound-state (electron) density was fully incorporated into the cross sections (2) in order to analyze the satellite excitations following the $4 d$ photoionization. These satellites arise mainly because of the rearrangement of the electron density in the course of photoionization that gives rise to a finite shakeup probability for the $5 s$ valence electron. In the MCDF method, this rearrangement is taken into account by properly including the overlap of the one-electron orbitals from the ground and final states in the evaluation of the photoionization amplitudes [36].

As discussed below, the double ionization of cadmium proceeds mainly via a subsequent Auger emission, leading to the $4 d^{10}$ ground state of $\mathrm{Cd}^{2+}$ and several low-lying $4 d^{9} n l_{j}$ levels. The corresponding Auger amplitudes arise from the coupling of the hole states to the one-electron continuum of the next higher charge state. These amplitudes and all the associated decay rates were calculated by means of the AUGER component of the RATIP program [34], in which the continuum spinors are solved within a spherical but leveldependent potential of the final ion. This procedure also includes the exchange interaction of the emitted electron with the bound-state density; see Refs. [30,37] for further details with regard to the construction of the scattering states and the use of the matrix elements. However, no relaxation has been included in the Auger calculations to keep them feasible for the large number of intermediate and final states.

Although for complex shell structures, such as neutral or singly ionized cadmium, not all details can be reproduced by the computations, they helped identify most of the ionization paths. Below, we make use of the reference configuration in order to explain the ionization dynamics through the photoionization of a $4 d$ electron, accompanied by valenceshell excitation, and subsequent Auger emission.

\section{RESULTS AND DISCUSSION}

Figure 1 shows spectra of doubly ionized cadmium recorded at the photon energies 40.814 and $48.37 \mathrm{eV}$. The ground state of the dication, $4 d^{10}{ }^{1} S_{0}$ is found to have an ionization energy of $25.91 \pm 0.04 \mathrm{eV}$, which corresponds to the maximum of the first most intense peak in Fig. 1. This is in good agreement with the values given in Moore's tables [38]. Weak peaks discernible at lower binding energies in both spectra are attributed to artifacts related to the shape of the light pulse from the helium lamp. Because the artifacts derive from a fixed time difference they are most apparent for low ionization energies, where the electron flight times are short, and they will give rise only to asymmetry in the observed line shapes at higher ionization energies. A faint signal at $\sim 30 \mathrm{eV}$ ionization energy can be observed in the spectrum recorded at $48.37 \mathrm{eV}$, which is most likely caused by afterglow of the helium lamp.

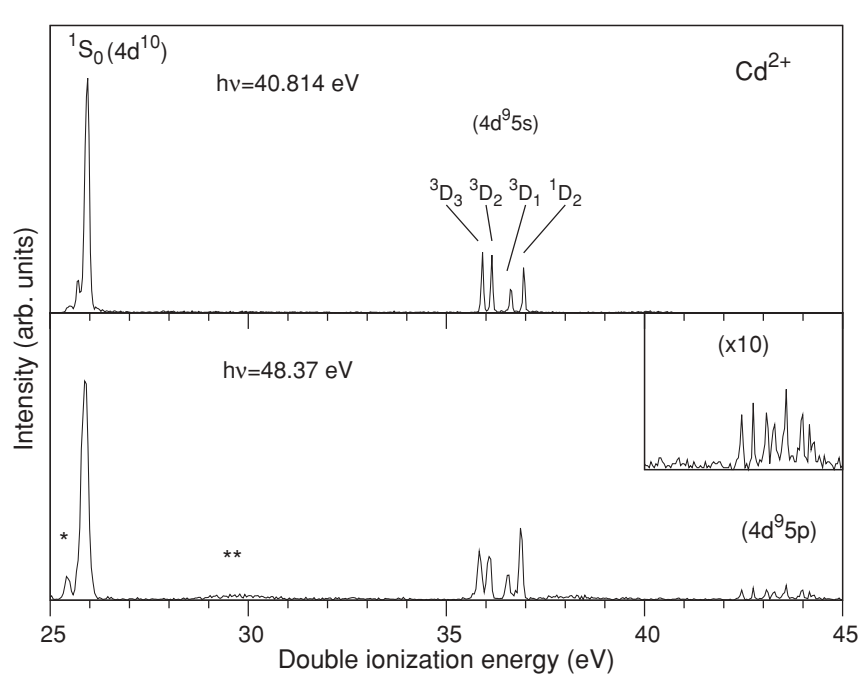

FIG. 1. Single-photon double-ionization spectra of cadmium recorded at 40.814 (upper panel) and 48.37 (lower panel) eV. The light source causes some artificial structure to appear in the spectra, as discussed in the text. This is indicated by single and double asterisks, respectively.

At higher ionization energies, we resolve the ${ }^{3} D_{3},{ }^{3} D_{2}$, ${ }^{3} D_{1}$, and ${ }^{1} D_{2}$ levels of the $4 d^{9} 5 s$ configuration. Furthermore, we also partly resolve the levels of the $4 d^{9} 5 p$ configuration, which, like the $4 d^{9} 5 s$ levels, are known from optical spectroscopy $[38,39]$. The ground state and first excited states in the cadmium double photoionization spectrum measured at the He photon energies closely resemble the corresponding states in mercury, which were investigated in a previous work by some of us [27]. In that study it was pointed out that the ground state is by far the most populated state, in contrast to the situation in single ionization, where the peak for the ground state is comparatively weak. As we can see from Fig. 1, this is the case also for cadmium.

The relative intensities within the $4 d^{9} 5 s$ states are found to be 2.1:1.9:1:1.6 at $40.814 \mathrm{eV}$ and 2.1:2.0:1:2.6 at $48.37 \mathrm{eV}$ photon energy. The favoring of singlet $\left({ }^{1} D\right)$ compared with triplet states with increasing photon energy has been found earlier for $\mathrm{Ne}$ [40], where it was attributed to changes in the relative contributions from direct and indirect ionization. In the present case no significant contribution from direct ionization is found at either photon energy. The change in the relative intensities observed may therefore more likely be related to the available intermediate states at the two photon energies, and the branching ratios of their decay.

In Fig. 2 double ionization spectra of Cd recorded at 85.1and $200 \mathrm{eV}$ photon energies are shown. The dominating feature at both photon energies is an intense band centered at $\sim 50 \mathrm{eV}$ ionization energy. In the spectrum recorded at $85.1 \mathrm{eV}$ photon energy this band is resolved into four peaks, with ionization energies of 48.1, 48.8, 49.7 and $50.8 \mathrm{eV}$, respectively, which are in good agreement with the known energies of the ${ }^{3} F_{4}$, ${ }^{3} F_{3,2},{ }^{3} P_{2}$, and ${ }^{1} G_{4}$ states of the $4 d^{8} s^{2}$ configuration $[38,39]$. A weaker peak is found at an ionization energy of $54.4 \mathrm{eV}$, which fits the energy of the ${ }^{1} S_{0}$ state, as known from optical spectroscopy [38]. Two shoulders are seen on the high-energy side of the ${ }^{1} G_{4}$ peak that are not assignable to any $4 d^{8} 5 s^{2}$ state. 


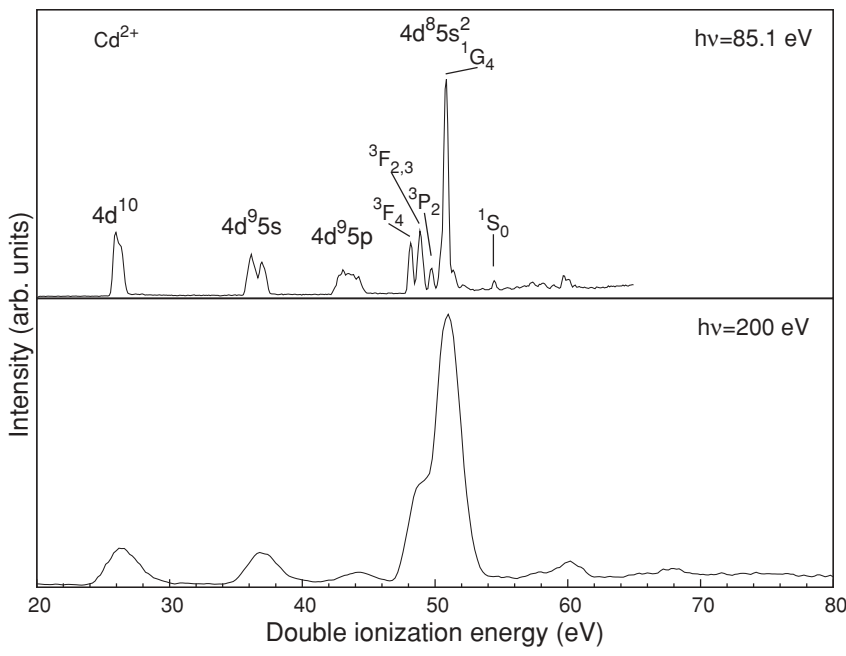

FIG. 2. Single-photon double-ionization spectra of cadmium recorded at 85.1 (upper panel) and 200 (lower panel) eV. The expected peaks for the ${ }^{3} P_{1,0}$ and ${ }^{1} D_{2}$ states of the $4 d^{8} s^{2}$ configuration are not resolved from the ${ }^{1} G_{4}$ peak but contribute to a shoulder on its low-energy side.

They most likely result from $4 d^{9} 6 s$ or $4 d^{9} 5 d$ configurations. However, due to the limited resolution of the experiment, in particular with respect to the density of the states involved, we refrain from a detailed assignment of these features.

The relative intensities of the $4 d^{8} 5 s^{2}$ states merit some discussion. Adopting the labels used by Kleef et al. we find the intensity ratio between the ${ }^{3} F_{4},{ }^{3} F_{3,2},{ }^{3} P_{2}$, and ${ }^{1} S_{0}$ states to be approximately 8:12:4:1, where in the ${ }^{1} S_{0}$ case the error may be quite high due to a nonlinearity in the background. Nevertheless, the relative intensities between these states does not differ much from their statistical weights of 9:12:5:1. The relative intensity of the sum of the ${ }^{3} P_{1,0}$ and ${ }^{1} D_{2}$ (unresolved) and ${ }^{1} G_{4}$ states to the ${ }^{3} F$ states is approximately 2 , which is more than twice that of the statistically expected value.

In the spectrum recorded at $200 \mathrm{eV}$ photon energy the $4 d^{8} 5 s^{2}$ states are also very prominent. At this photon energy we no longer resolve individual states of this configuration but observe a structured band. From the shape of the band it is clear that the ${ }^{1} G_{4}$ state is still strongly favored compared to the ${ }^{3} F$ states.

At approximately 60- and $68 \mathrm{eV}$ ionization energy two additional features are seen. A large number of $\mathrm{Cd}^{2+}$ states, based on $4 d^{9} n l$ and $4 d^{8} n l n^{\prime} l^{\prime}$ configurations, are likely to be present in this region. Because of the non-neglible intensity of these features they are possibly related to $4 d^{-2}$ ionization, which is expected to be influenced by Coster-Kronig decay from $4 s$ and $4 p$ holes. A more detailed assignment would require a much extended computation, including interchannel interactions, which is beyond the scope of this work. In addition, Coster-Kronig decay from $4 s$ to $4 p^{-1} 4 d^{-1}$ is visible at approximately $110 \mathrm{eV}$ ionization energy (but not shown in Fig. 2) as a broad ( $\sim 20 \mathrm{eV}$ full width) band with some slight intensity modulations. We will discuss this decay further below in the context of Fig. 5, which shows this energy region of the spectrum. Apart from this feature no clearly discernible structure above the smooth background is observed at higher ionization energies.
From analysis of the two-dimensional coincidence data one can obtain detailed information on double-ionization processes. For example, in direct double ionization, where the two electrons are ejected simultaneously, the electron energy distribution will be continuous because the two electrons can share the excess energy in an arbitrary fashion. In contrast, if an intermediate state is involved, the electron energies will be fixed but may be broadened according to the widths of intermediate and/or final states. It should also be noted that a large number of close-lying discrete states combined with a finite electron energy resolution can make electron energy distributions appear continuous even though the doubleionization process itself is indirect. This is well known for the double ionization of molecules, where close-lying vibrational levels can contribute to spectral congestion, whereas in atoms the situation is usually much clearer.

In Fig. 3 the double-ionization data of $\mathrm{Cd}$ recorded at $85.1 \mathrm{eV}$ are represented in form of a coincidence map with both electron kinetic energies plotted on the vertical axis versus the ionization energy plotted on the horizontal axis. To emphasize the connection to the previous discussion we include the double-ionization final-state spectrum again in the upper panel of this figure.

From the coincidence map it can be seen that the electrons ejected in formation of the states primarily associated with the $4 d^{10}, 4 d^{9} 5 s$, and $4 d^{9} 5 p$ configurations predominantly have fixed energies, which indicates an indirect double ionization mechanism involving intermediate excited states of $\mathrm{Cd}^{+}$. There is also only little evidence for the direct process in the coincidence data recorded at lower photon energies (not

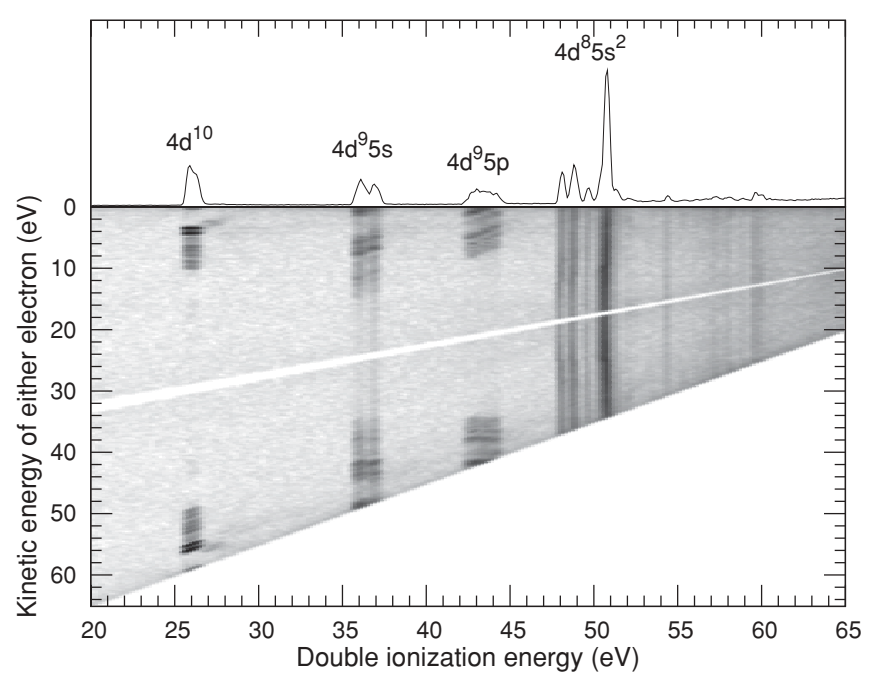

FIG. 3. Coincidence map (lower panel) where the electron energy ( $y$ axis) of either electron is plotted versus ionization energy ( $x$ axis) of the two electrons ejected on single-photon ionization at $85.1 \mathrm{eV}$ photon energy. Note the linear islands of intensity connected with formation of the $4 d^{9} 5 s$ and $4 d^{9} 5 p$ states; when an intermediate state decays to several final states the photoelectron will be fixed in energy while the Auger electron energy varies, causing the observed slope at low electron energies. At equal electron energy there is a gap in intensity because of the $\sim 10$-ns detection dead time in the experiment. The intensity in the map is in arbitrary units and has been scaled by taking its square root in order to enhance weak features. 


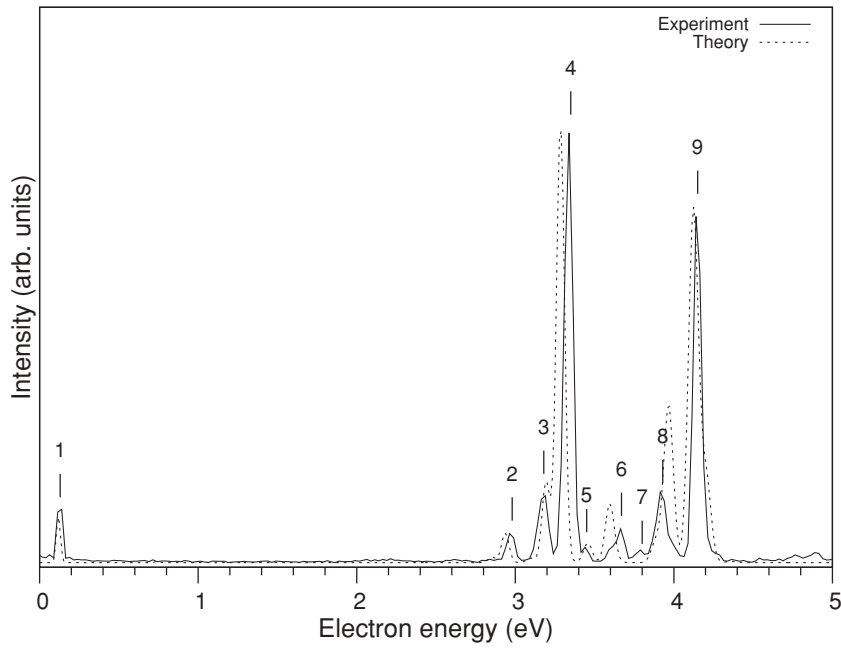

FIG. 4. (Solid line) Energy distribution of electrons ejected in the formation of the $\mathrm{Cd}^{2+}$ ground state. (Dotted line) A simulated spectrum based on single ionization cross sections calculated in this work, which were broadened by the experimental resolution and normalized in intensity to the experimental spectrum.

shown). The kinetic energy distribution of electrons forming the ${ }^{1} S_{0}$ dication ground state is, in particular, dominated by several strong features below $5 \mathrm{eV}$. Some structures are visible also at higher electron energies, but the intensity drops drastically at approximately $10 \mathrm{eV}$ electron energy to the extent that no structures are apparent above the noise level. The intensity cutoff occurs at the binding energy of the states related to the $4 d^{9} 5 s$ configuration. This suggests photoionization to $4 d^{9} n l n^{\prime} l^{\prime}$ correlation satellite states followed by Auger decay to $4 d^{10}$ as the major double-ionization mechanism for formation of the $\mathrm{Cd}^{2+}$ ground state. In order to confirm this finding and identify the most prominent peaks we have calculated the photoionization cross section of the singly ionized but doubly excited $4 d^{9} 5 s n l$ states lying $<5 \mathrm{eV}$ above the double-ionization threshold. Above $5 \mathrm{eV}$, the density of possible doubly excited states becomes too large and does not allow for identification of individual peaks.

In Fig. 4 a simulated spectrum based on our numerical results is compared with the experimental spectrum recorded at $85.1 \mathrm{eV}$. A constant shift of $0.8 \mathrm{eV}$ has been added to the calculated energies to compensate for missing correlation energy. After this shift is introduced the agreement is generally good, as can be seen. However, some deviations remain, as a result of the limited account of electron-electron correlation in both the neutral and singly ionized atom, which is necessary in order to keep the calculations of the photoionization cross sections feasible. Possibly also branching into radiative transitions, which are not detected in the experiment, could change the relative intensities of the simulated spectrum compared to the observed one.

In contrast to the double ionization of mercury, which has a homologous valence configuration $\left(5 d^{10} 6 s^{2}\right)$ to that of $\mathrm{Cd}$, the most prominent autoionization peaks connected to the groundstate configuration appears to result from "normal" $4 d^{-1} n l$ correlation satellites, whereas in mercury a strong contribution from three-hole states arising from the $5 d^{9} 6 p^{2}$ configuration
TABLE I. Energies and relative intensities of the most prominent peaks found in the formation spectrum of the dication ground state of Cd. The peak labeling follows Fig. 4. By comparing with our calculations, designation in LSJ notation and percentage of leading configuration is given (where possible) of the level of $\mathrm{Cd}^{+}$associated with the peak. Peaks 4 and 9 were previously classified by Mansfield and Murnane [19].

\begin{tabular}{lcccc}
\hline \hline Peak no. & Expt. (eV) & Rel. int. & Design. & Leading config. \\
\hline 1 & 0.13 & 2.5 & ${ }^{2} P_{1 / 2}^{\mathrm{o}}$ & $4 d^{9} 5 s 5 p(77)$ \\
2 & 2.98 & 1.6 & ${ }^{4} F_{5 / 2}$ & $4 d^{9} 5 s 5 d(30)$ \\
3 & 3.18 & 4.9 & ${ }^{2} P_{3 / 2}$ & $4 d^{9} 5 s 5 d(59)$ \\
4 & 3.34 & 25.3 & ${ }^{2} D_{5 / 2}$ & $4 d^{9} 5 s 5 d(61)$ \\
5 & 3.45 & $<1$ & ${ }^{4} G_{5 / 2} /{ }^{4} D_{3 / 2}$ & $4 d^{9} 5 s 5 d$ \\
6 & 3.66 & 2.4 & ${ }^{4} F_{3 / 2}$ & $4 d^{9} 5 s 5 d(47)$ \\
7 & 3.80 & $<1$ & - & - \\
8 & 3.93 & 5.7 & ${ }^{2} P_{3 / 2} /{ }^{2} D_{5 / 2}$ & $4 d^{9} 5 s 5 d$ \\
9 & 4.16 & 20.9 & ${ }^{2} D_{3 / 2}$ & $4 d^{9} 5 s 5 d(52)$ \\
& $4.4-10$ & 28 & - & - \\
\hline \hline
\end{tabular}

was found [27] due to configuration interaction in the neutral species [41]. Energies and assignments of the identified $\mathrm{Cd}^{+}$ intermediate states are given in Table I.

Formation of the excited dicationic states with leading configurations $4 d^{9} 5 s$ and $4 d^{9} 5 p$ is accompanied by complex electron energy distributions. A detailed identification and assignment of individual states is not possible in this case and therefore the formation of these states will be discussed primarily qualitatively. We note in Fig. 3 that the intermediate $\mathrm{Cd}^{+}$states which decay to the $4 d^{9} 5 s$ and $4 d^{9} 5 p$ states are not observed in transitions to the ground state although they would be energetically possible. There is, however, an intensity increase at some of the same intermediate $\mathrm{Cd}^{+}$energies, which suggests that the same excited states can decay to both the $4 d^{9} 5 s$ and $4 d^{9} 5 p$ states. At higher electron energies a continuous distribution is found, and while we cannot rule out a contribution from direct double ionization, the intensity comes to a large extent from Coster-Kronig decay following $4 p$ photo ionization, which we will discuss further below. A broad band of window resonances in the photoabsorption spectrum of $\mathrm{Cd}^{+}$at energies $27-40 \mathrm{eV}$ above the $\mathrm{Cd}^{+}$ionization energy has been observed by Kilbane et al. [6], where the resonances below $33 \mathrm{eV}$ were tentatively assigned to states of $4 d^{9} 5 p 5 d$ or $4 d^{8} 5 s^{2} 5 p$ configurations. States with similar $4 d^{9} n \ln ^{\prime} l^{\prime}$ or $4 d^{8} 5 s^{2} n l$ configurations are expected to be the $\mathrm{Cd}^{+}$states which decay to the $4 d^{9} 5 s$ and $4 d^{9} 5 p$ configurations. From an orbital picture it is difficult to explain why $4 d^{9} n l n^{\prime} l^{\prime} \mathrm{Cd}^{+}$ states should not undergo Auger decay to the $\mathrm{Cd}^{2+}$ ground state. However, an Auger decay from $4 d^{8} 5 s^{2} n l$ to the ground state would involve a rearrangement of two bound electrons and hence is less likely. In view of this we suggest that a major channel for population of the $4 d^{9} 5 s$ and $4 d^{9} 5 p$ states involves $4 d^{8} 5 s^{2} n l$ states. Our calculations confirm the importance of intermediate $\mathrm{Cd}^{+}$double-hole states based on a $4 d^{8}$ core configuration for the formation of the first excited states of $\mathrm{Cd}^{2+}$ but because of the complexity of the shell structures involved no attempt was made to reproduce the details of the experimental formation spectrum. 
TABLE II. $4 s$ and $4 p$ binding energies in $\mathrm{eV}$. The value for $4 p$ is taken at the half-maximum of the leading edge of the observed peak structure.

\begin{tabular}{cc}
\hline \hline $4 s$ & $4 p$ \\
\hline $115.9(3)$ & $70.5(5)$ \\
\hline \hline
\end{tabular}

As expected, the double-ionization spectra recorded above the $4 p$ and $4 s$ ionization thresholds (cf. Table II) are strongly influenced by Coster-Kronig (CK) transitions. This is perhaps most clearly seen by the intensity of the $4 d^{8} 5 s^{2}$ states, which are mainly populated by super-CK decay of a $4 p$ hole. In our coincidence experiment the different decay channels of a $4 p$ or $4 s$ hole, respectively, can be studied. In the data recorded at $85.1 \mathrm{eV}$ photon energy we cannot, however, separate the $4 p$ photoelectron from the CK electron due to the large lifetime width of the $4 p$ hole and the proximity of the photon energy to the $4 p$ threshold. To resolve this issue we recorded coincidence data at $200 \mathrm{eV}$ photon energy, which is shown in Fig 5. At this photon energy the distinction between photo- and CK electrons are unambiguous.

The main decay channels for the $4 s$ hole are expected to involve states of the $4 p^{-1} 4 d^{-1}, 4 d^{-2}$ and $4 d^{-1} 5 s^{-1}$ configurations [42], which is confirmed by observation of the $4 s$ photoelectron, identified by the $\sim 7 \mathrm{eV}$ broad band at around $85 \mathrm{eV}$ electron kinetic energy, as observed in the electron distributions of the states mentioned. The $4 p^{-1} 4 d^{-1}$ states created in this way can be expected to rapidly undergo CosterKronig decay leading to $4 d^{-3}$ states [11], which is confirmed by preliminary analysis of the triple electron coincidences that are also recorded in our experiment. That the $4 p^{-1} 4 d^{-1}$ states are observed in the set of two-electron coincidences as well as three-electron coincidences is likely a consequence of the $\sim 50 \%$ collection-detection efficiency of our electron spectrometer. The intensity of the peak structure at around $110 \mathrm{eV}$ ionization energy in Fig. 5 should therefore not be taken as a true measure of the strength of the $4 s^{-1} \rightarrow 4 p^{-1} 4 d^{-1}$ transition, nor should the peak shape be taken at face value.

We could not detect decay of $4 s$ to the $\mathrm{Cd}^{2+}$ ground state and only very little intensity is observed in the $4 d^{9} 5 p$ states. This is also the case for the $4 p$ hole where decay to the $4 d^{-2}$ states is by far the most intense, but some intensity is also seen in the $4 d^{9} 5 s$ states. This behavior explains the observed change in intensity between the $4 d^{9} 5 s$ and $4 d^{9} 5 p$ doubly ionized states at the two photon energies; the peaks related to the $4 d^{9} 5 p$ states are approximately 2.9 times weaker relative to the $4 d^{9} 5 \mathrm{~s}$ peaks when going from 85.1 - to $200 \mathrm{eV}$ photon energy. Interestingly the $\mathrm{Cd}^{2+}$ ground state and $4 d^{9} 5 p$ states appear to be populated mainly via essentially the same intermediate states at 85.1and $200 \mathrm{eV}$ photon energy. As the ground state is much more strongly populated at the higher photon energy, the photon energy dependence on ionization of the intermediate states must differ markedly.

Although the $4 d^{8} 5 s^{2}$ states are not fully resolved at 200 $\mathrm{eV}$ photon energy it is clear from Fig. 5 that both the

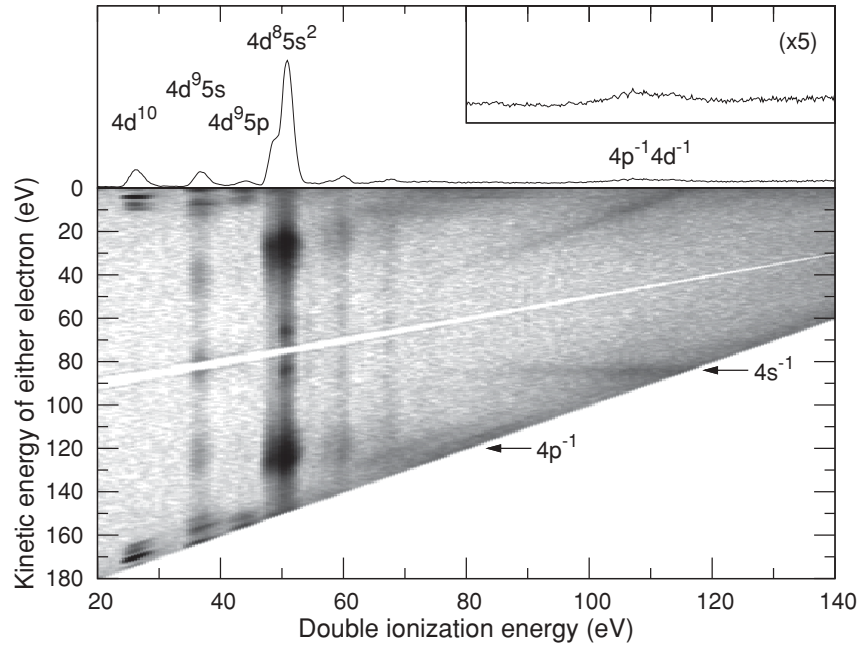

FIG. 5. Coincidence data recorded at $200 \mathrm{eV}$ photon energy, represented in a similar way as the data obtained at $85.1 \mathrm{eV}$ photon energy, which is shown in Fig. 3. The approximate kinetic energies (vertical axis) at which $4 s$ and $4 p$ photoelectrons appear are indicated by arrows in the figure.

$4 s$ and $4 p$ holes preferentially decay to the ${ }^{1} G_{4}$ state as opposed to the ${ }^{3} F$ states. This is observed especially for the decay of the $4 s$ hole, which is seen in the absence of the $4 s$ photoelectron on the low-energy side of the band associated with the $4 d^{8} 5 s^{2}$ configuration.

\section{CONCLUSIONS}

We have measured the double-ionization spectrum of cadmium at 40.814-, 48.37-, 85.1-, and $200 \mathrm{eV}$ photon energy and found that the $\mathrm{Cd}^{2+}$ states are mainly populated indirectly, with strong channel selectivity for some of the states studied. In particular the $\mathrm{Cd}^{2+}$ ground state appears to always be reached through the same excited $\mathrm{Cd}^{+}$states in this energy range, despite the opening of various inner shells at the higher photon energies. Several of these intermediate states have been identified. At higher excitation energies Coster-Kronig processes become increasingly important at photon energies above the $4 p$ ionization threshold.

\section{ACKNOWLEDGMENTS}

This work has been financially supported by the Swedish Research Council (VR), the Göran Gustafsson Foundation (UU/KTH), and the Knut and Alice Wallenberg Foundation, Sweden. This work was also supported by the European Community - Research Infrastructure Action under the FP6 "Structuring the European Research Area" Programme (through the Integrated Infrastructure Initiative "Integrating Activity on Synchrotron and Free Electron Laser Science," Contract R II 3-CT-2004-506008). S.F. acknowledges the support by the FiDiPro programme of the Finnish Academy.
[1] R. B. Cairns, H. Harrison, and R. I. Schoen, J. Chem. Phys. 51, 5440 (1969).
[2] J. Berkowitz and C. Lifshitz, J. Phys. B 1, 438 (1968).

[3] S. Süzer and D. A. Shirley, J. Chem. Phys. 61, 2481 (1974). 
[4] J. E. Hansen, Phys. Rev. A 15, 810 (1977).

[5] M. W. D. Mansfield, Proc. R. Soc. London 362, 129 (1978).

[6] D. Kilbane, E. T. Kennedy, J.-P. Mosnier, J. T. Costello, and P. van Kampen, J. Phys. B 38, 83 (2005).

[7] M. Kutzner, C. Tidwell, S. E. Vance, and V. Radojević, Phys. Rev. A 49, 300 (1994).

[8] P. H. Kobrin, U. Becker, S. Southworth, C. M. Truesdale, D. W. Lindle, and D. A. Shirley, Phys. Rev. A 26, 842 (1982).

[9] J. Jiménez-Mier, C. D. Caldwell, and M. O. Krause, Phys. Rev. A 39, 95 (1989).

[10] S. P. Kowalczyk, L. Ley, R. L. Martin, F. R. McFeely, and D. A. Shirley, Faraday Discuss. Chem. Soc. 60, 7 (1975).

[11] M. Ohno and J. M. Mariot, J. Phys. C 14, L1133 (1981).

[12] S. Svensson, N. Mårtensson, E. Basilier, P. Å. Malmquist, U. Gelius, and K. Siegbahn, Phys. Scr. 14, 141 (1976).

[13] U. Gelius, J. Elec. Spec. Rel. Phen. 5, 985 (1974).

[14] R. E. LaVilla, Phys. Rev. A 17, 1018 (1978).

[15] S. Lundqvist and G. Wendin, J. Elec. Spec. Rel. Phen. 5, 513 (1974).

[16] G. Wendin and M. Ohno, Phys. Scr. 14, 148 (1976).

[17] M. Ohno, J. Elec. Spec. Rel. Phen. 131-132, 3 (2003).

[18] D. M. P. Holland, K. Codling, and J. B. West, J. Phys. B 15, 1473 (1982).

[19] M. W. D. Mansfield and M. M. Murnane, J. Phys. B 18, 4223 (1985).

[20] V. Pejčev, K. J. Ross, D. Rassi, and T. W. Ottley, J. Phys. B 10, 459 (1977).

[21] J. H. Eland, Adv. Chem. Phys. 141, 103 (2009).

[22] J. H. D. Eland, O. Vieuxmaire, T. Kinugawa, P. Lablanquie, R. I. Hall, and F. Penent, Phys. Rev. Lett. 90, 053003 (2003).

[23] D. Batchelor, R. Follath, and D. Schmeisser, Nucl. Instrum. Methods A 467-468, 470 (2001).

[24] J. H. D. Eland, P. Linusson, L. Hedin, E. Andersson, J.-E. Rubensson, and R. Feifel, Phys. Rev. A 78, 063423 (2008).
[25] R. Feifel, J. H. D. Eland, L. Storchi, and F. Tarantelli, J. Chem. Phys. 125, 194318 (2006).

[26] J. H. D. Eland and R. Feifel, Chem. Phys. 327, 85 (2006).

[27] J. H. D. Eland, R. Feifel, and D. Edvardsson, J. Phys. Chem. A 108, 9721 (2004).

[28] T. X. Carroll, J. D. Bozek, E. Kukk, V. Myrseth, L. J. Sæthre, T. D. Thomas, and K. Wiesner, J. Elec. Spec. Rel. Phen. 125, 127 (2002).

[29] O. Edqvist, E. Lindholm, L. E. Selin, and L. Åsbrink, Phys. Scr. 1, 25 (1970).

[30] S. Fritzsche, Phys. Scr. T 100, 37 (2002).

[31] I. P. Grant, Methods in Computational Chemistry, edited by S. Wilson (Plenum Press, New York, 1988).

[32] S. Fritzsche, C. F. Fischer, and G. Gaigalas, Comput. Phys. Commun. 148, 103 (2002).

[33] F. A. Parpia, C. F. Fischer, and I. P. Grant, Comput. Phys. Commun. 94, 249 (1996).

[34] S. Fritzsche, J. Elec. Spec. Rel. Phen. 114-116, 1155 (2001).

[35] G. Gaigalas, T. Zalandauskas, and S. Fritzsche, Comput. Phys. Commun. 157, 239 (2004).

[36] S. Fritzsche, A. Surzhykov, and T. Stöhlker, Phys. Rev. A 72, 012704 (2005).

[37] S. Fritzsche, B. Fricke, and W.-D. Sepp, Phys. Rev. A 45, 1465 (1992).

[38] C. E. Moore, Atomic Energy Levels, Vol. 3 (US GPO, Washington, DC, 1958).

[39] T. A. M. van Kleef, Y. N. Joshi, and P. Uijlings, Phys. Scr. 22, 353 (1980).

[40] T. Kaneyasu, Y. Hikosaka, E. Shigemasa, F. Penent, P. Lablanquie, T. Aoto, and K. Ito, Phys. Rev. A 76, 012717 (2007).

[41] J. Berkowitz, J. L. Dehmer, Y. K. Kim, and J. P. Desclaux, J. Chem. Phys. 61, 2556 (1974).

[42] V. Schmidt, Rep. Prog. Phys. 55, 1483 (1992). 